\title{
BMJ Open Impact of a work-based feedback intervention on student performance during clinical placements in acute-care healthcare settings: a quasi- experimental protocol for the REMARK programme
}

Christine Ossenberg (D) ,1,2 Marion Mitchell,, ${ }^{1,3}$ Amanda Henderson ${ }^{2}$

To cite: Ossenberg $\mathrm{C}$, Mitchell M, Henderson A. Impact of a work-based feedback intervention on student performance during clinical placements in acute-care healthcare settings: a quasiexperimental protocol for the REMARK programme. BMJ Open 2020;10:e034945. doi:10.1136/ bmjopen-2019-034945

- Prepublication history and additional material for this paper are available online. To view these files, please visit the journal online (http://dx.doi. org/10.1136/bmjopen-2019034945).

Received 16 0ctober 2019 Revised 19 February 2020 Accepted 29 April 2020

\section{Check for updates}

(C) Author(s) (or their employer(s)) 2020. Re-use permitted under CC BY-NC. No commercial re-use. See rights and permissions. Published by BMJ.

${ }^{1}$ School of Nursing and Midwifery, Griffith University, Nathan, Queensland, Australia ${ }^{2}$ Nursing Practice Development Unit, Princess Alexandra Hospital, Woolloongabba, Queensland, Australia ${ }^{3}$ Intensive Care, Princess Alexandra Hospital, Brisbane, Queensland, Australia

Correspondence to Christine 0ssenberg; christine.ossenberg@griffithuni. edu.au

\section{ABSTRACT}

Introduction Current perspectives present feedback as a dynamic, dialogic process. It is widely accepted that feedback can have an impact on workplace performance, however, how dialogic feedback is enacted with the learner in authentic healthcare settings is less apparent. This paper seeks to describe the design and development of an implementation study to promote the learner voice in the feedback process and improve feedback encounters between learners and learning partners in healthcare settings.

Methods and analysis A quasi-experimental study design will be used to evaluate whether implementation of a work-based intervention to improve feedback impacts student performance during clinical placements in healthcare settings. Student performance will be measured at three time points: baseline (pre), midplacement (post-test 1) and end-placement (post-test 2) in keeping with standard assessment processes of the participating university. The intervention is underpinned by Normalisation Process Theory and involves a layered design that targets learners and learning partners using best-practice education strategies. Data regarding participants' engagement with feedback during clinical placements and participants' level of adoption of the intervention will be collected at the completion of the clinical placement period.

Ethics and dissemination This study has ethics approval from both Griffith University and Metro South Health Human Research and Ethics committees. Dissemination of results will be local, national and international through forums, seminars, conferences and publications.

\section{INTRODUCTION}

Much has been written about feedback; its purpose, application and effectiveness. Current insights embrace feedback as a dialogic, ${ }^{1}$ socio-constructivist process. $^{2-4}$ Accordingly, this stance shifts our lens from the mere transmission of information from expert to novice, to a shared

\section{Strengths and limitations of this study}

- This protocol uses Normalisation Process Theory in the design, implementation and evaluation of the study.

- This protocol is an example of the use of the normalising process to optimise the adoption of evidence into routine practice.

- This methodology uniquely engages all groups involved in the feedback process during a student's clinical placement in the healthcare setting.

- This protocol uses a quasi-experimental design which may make it susceptible to threats to the internal validity of the study.

approach between learner (ie, the person who actively engages in and even directs the feedback process) and learning partner (ie, someone who supports a learner in the feedback process) where feedback is dynamic and co-created. ${ }^{5}$ The learner's or learning partner's control in 'driving' the feedback process changes depending on the situation, existing skills and experience and assessment requirements. Embedding opportunities for formative feedback ${ }^{6}$ are essential to guide the learner's progress as well as the learning partner's and is assisted through timeliness of feedback, negotiation of learning goals and explicit evaluation criteria/standards.

Feedback that enhances performance in the workplace is critical for the development of the broad elements of professional practice to ensure standards of practice and patient safety are met. Not unlike academic based assessment (eg, assignments and exams), workplace-based assessment has some nonnegotiable criteria-whether that is a specific skill/knowledge to be demonstrated or 
evidence of the learner meeting established professional practice standards. This does not render the dialogic feedback process irrelevant, on the contrary, processes required to support the learner in achieving these criteria and feedback required for learner growth can and should be mutually negotiated.

While the value of dialogic feedback is apparent there is minimal evidence of its use with students in healthcare settings. Literature pertaining to learning in clinical settings recognises that student views, thoughts or opinions are not readily sought within these traditional contexts. ${ }^{7}$

This paper provides a protocol for a work-based intervention specifically designed to shift traditions in the delivery of feedback in clinical contexts.

\section{Theoretical framework}

Literature on implementation research has expanded over the last two decades and highlights an increase in the use of theory within implementation studies. However, theory frequently remains underused or misused. ${ }^{8}$ Implementation is considered '...the process of putting to use or integrating evidence-based interventions within a setting ${ }^{9}$ (p118). Frameworks and theories are important to provide better understanding and explanation of the mechanisms by which implementation is more likely to do well and ways to address assorted challenges in modifying practice. ${ }^{1011}$ Additionally, theories and frameworks provide an explicit design on which research can be developed, applied and evaluated. ${ }^{12}$

A diverse range of theories and frameworks are available for use in implementation studies. ${ }^{13}$ These frameworks vary in the flexibility of constructs (broad to operational), focus of activities (dissemination and/or implementation) and the level of which the application operates (system, organisation, individual). ${ }^{13}$ Selection of a theoretical framework for this study was influenced by the focus of the research activities (implementation) and level at which the application was focussed (organisational and individual). Taking these factors into consideration, the development and implementation of this intervention is underpinned by Normalisation Process Theory (NPT).

NPT is a sociological theory of action offering insights into how people interact, organise and perform the work collectively. ${ }^{14}$ It explores social processes and facilitates the explanation of how material practices (eg, complex interventions) become routine in everyday practice (normalised). Work is defined as the purposive social action that involves the investment of personal and group resources to achieve goals ${ }^{15}$ (p539). The theory concentrates on three core problems: implementation (bringing practices into action), embedding (process in which practices become or do not become routine) and integration (practices are reproduced and sustained) ${ }^{16}{ }^{17}$ While NPT has primarily been used to evaluate the implementation of complex interventions, literature using the theory to develop an intervention and optimise implementation is emerging. ${ }^{18-21}$

As a middle-range theory-one which addresses the distinct question of how practices become implemented and routinely embedded ${ }^{15}{ }^{22}$-NPT is built around four principal constructs referred to as generative mechanisms: ${ }^{15}$

1. Coherence - individual and communal meaning and sense-making that participants invest to promote or inhibit a practice;

2. Cognitive participation - the commitment and engagement participants invest to adopt and legitimise the practice;

3. Collective action - the effort and everyday practices participants invest to enact the work and make it function; and

4. Reflexive monitoring-individual and communal comprehension and appraisal of the effects of the practice.

Each of the overarching mechanisms contain components that further explicate the immediate and organising work of an implementation process (table 1). Collectively, these can serve as a sensitising tool for researchers when considering or planning implementation research. ${ }^{1821}$

Table 1 Schema of Normalisation Process Theory mechanisms, investments and components

\begin{tabular}{|c|c|c|c|}
\hline \multirow[b]{2}{*}{ Mechanism } & \multirow[b]{2}{*}{ Investment } & \multicolumn{2}{|l|}{ Components } \\
\hline & & Immediate work & Organising work \\
\hline Coherence & Meaning & $\begin{array}{l}\text { Differentiation } \\
\text { - Individual specification }\end{array}$ & $\begin{array}{l}\text { Internalisation } \\
\text { Communal specification }\end{array}$ \\
\hline Cognitive participation & Commitment & $\begin{array}{l}\text { - Initiation } \\
\text { - Legitimation }\end{array}$ & $\begin{array}{l}\text { Enrolment } \\
\text { - Activation }\end{array}$ \\
\hline Reflexive action & Comprehension & $\begin{array}{l}\text { - Systematisation } \\
\text { - Individual appraisal }\end{array}$ & $\begin{array}{l}\text { - Reconfiguration } \\
\text { - Communal appraisal }\end{array}$ \\
\hline
\end{tabular}

${ }^{*}$ Adapted from May and Finch, 2009. 
The limited scope and conceptual range assist in making NPT practically workable in advancing practice. ${ }^{14}$ As a sociological theory, NPT aligns with current socioconstructivist views of feedback. Furthermore, nurses and nursing (as with other health disciplines) occurs within a largely social context, involving interactions with patients, colleagues and the broader organisational environment. NPT is therefore an appropriate theory to explain the social processes that frame the implementation of a best practice feedback programme in workplace environments.

\section{Aim and objectives}

This study aims to examine the impact of a work-based educational intervention on student nurses' workplace performance. The intervention is derived from attributes that guide best practice for effective feedback and will be directed towards student nurses (referred to as students from here on), buddy nurses and clinical facilitators as they engage with each other during the student's clinical placement.

To address the aim of the study, the following question is proposed:

What impact does a work-based educational intervention drawing on the principles of effective feedback have on:

a. Student performance during clinical placements as measured by a validated assessment tool used nationally Australian Nursing Standards Assessment Tool (ANSAT)?

b. Student, buddy nurse and clinical facilitator engagement with the feedback process as measured by a feedback perception survey called the Quality Feedback Inventory (QFI)?

c. Student, buddy nurse and clinical facilitator adoption of best practice feedback principles as measured by the nuRse fEedback iMplementAtion framewoRK (REMARK) study survey?

A buddy nurse is a nurse who is assigned to work alongside students in the clinical unit/ward for a shift at a time. ${ }^{23}$ Clinical facilitators are nurses who have primary responsibility for working with and assesses a group of students during clinical placement. ${ }^{23}$

\section{METHODS AND ANALYSIS \\ Study design}

This study will be based on a quasi-experimental, nonequivalent control group design as randomisation is not practical due to the 'real-world' workforce demands of nursing. ${ }^{24}$ Furthermore, owing to the nature of the intervention, there will be no blinding to treatment condition for any participant or research team member.

Due to the dynamic nature of the healthcare setting, not all variables can be controlled for in applied research (eg, participant maturation), which can affect the internal validity of the design. ${ }^{25} \mathrm{~A}$ pre-assessment and post-assessment strategy for both control and intervention groups will be used. ${ }^{26}{ }^{27}$ In addition, participants in the control group will be geographically separated from participants in the intervention group, limiting diffusion or imitation of the intervention. ${ }^{25}$ Inclusion of these identified strategies to assuage threats to internal validity strengthens the study design. Outcome data from both groups will be collected using the same measures and at the same time points: pre-test (baseline), post-test 1 (midplacement assessment) and post-test 2 (end-placement assessment) (online supplementary file 1).

Patient and Public Involvement: Patients and the public will not be actively recruited to this study, nor will they actively inform the study.

\section{Setting}

The setting for this study will be two healthcare facilities within a large Hospital and Health Service in South East Queensland, Australia (control, Site A; intervention, Site B). Each facility, while slightly different bed capacity, has both inpatient and outpatient services. Both facilities are teaching hospitals providing educational opportunities for future healthcare professionals.

It is noted that the researcher team work at the control site, however, do not have any supervisory, assessment, reporting or management relationships with the students, nurses or clinical facilitators (CFs) at either the control or intervention site.

\section{Participants}

Participants will represent three groups: (i) students enrolled in the undergraduate Bachelor of Nursing at the participating university, (ii) buddy nurses employed by the healthcare facilities who support student learning in the clinical unit (usually on a shift-by-shift basis) during clinical placements at each site and (iii) CFs employed by the participating university who supervise and assess students during clinical placement at each site. Convenience sampling will be used and based on the availability of each population according to how clinical placements are organised. As no specific clinical area is targeted, participants from each population will reflect the diverse practice areas nursing care is undertaken and thereby aid in generalisation of the results. The researchers have no input into which site or unit the students, clinical facilitators and buddy nurses are allocated for clinical placements.

Only clinical placements of 4 weeks will be used to ensure sufficient time for implementation of the workbased intervention to have a measurable impact. Finalyear students are placed on two 4-week placement blocks during the study period. Students may therefore be placed at either the control and/or intervention sites for their placement blocks.

To be eligible for inclusion, participants will be: $\geq 18$ years of age, final year nursing students, buddy nurses who support final-year students in the clinical units at the healthcare facilities and university clinical facilitators who supervise and assess final year nursing students in the clinical units. Potential contamination will be reduced by only 
using the data from the student's first placement block in either the control or intervention site. Data collected from students who complete $\leq 50 \%$ of their placement will be excluded due to the lack of potential participation and engagement in the intervention. Students who attend their first placement at the intervention site will be excluded if they then attend the subsequent placement at the control site.

\section{Recruitment}

Explanatory statements outlining the project aims and details of what participation means for all potential participants will be distributed via existing organisation wide communication processes (eg, email) administered by senior nursing executives, nurse unit managers (NUMs) and clinical placement coordinators within each healthcare facility and university. Additional face-to-face opportunities at the healthcare facilities (eg, ward meetings) and university (eg, lecture sessions) will be held to inform potential participants of the study. The REMARK programme lead researcher will be available for potential participants to clarify the study information. Due to the structure of clinical placements at the healthcare facilities, recruitment of potential participants in each group will occur at the commencement of each clinical placement block. Collaboration between the clinical placement coordinators for the healthcare facilities and university and the REMARK programme lead researcher will be key in recruitment of the students, buddy nurses and CFs.

Participation is voluntary and participants are free to withdraw at any time without prejudice. Prospective informed written consent will be sought from the potential participants at the intervention site by the REMARK programme lead researcher before enrolment in the study. For those participants at the control site, the return of a partially or fully completed survey is accepted as an expression of consent to participate. It is anticipated that data collection will take 6 months.

\section{Study sites}

Control site

Assessment and supervision of student performance during clinical placement will be conducted by buddy nurses and CFs in accordance with the university's standard placement model requirements. The model involves the allocation of one CF to a group of six to eight students of the same year level. The CF has primary responsibility in the assessment of the student's performance. Students are allocated to multiple clinical units across the facility and therefore relies on students being assigned to a buddy nurse in the clinical unit on a shift-by-shift basis.

This model requires the completion of a mid-placement (formative) and end-placement (summative) assessment of student performance using the university's assessment tool-the ANSAT. Mid-placement assessment provides an opportunity for the student and CF to discuss current performance and opportunities for further progress; it is designed to be completed half-way through the clinical placement (for example, on a 4-week placement, midplacement assessment would occur at the end of the second week). End-placement assessment is completed at the end of the final week of clinical placement by the $\mathrm{CF}$ and is based on the student's performance over the duration of the clinical placement.

\section{Intervention site}

Assessment and supervision of student performance during clinical placement is the same as the control group with the addition of implementing a work-based intervention drawing on effective feedback principles during the students' clinical placement.

Historically the control and intervention sites have offered clinical placements for over 15 years at the participating healthcare facilities and are well acquainted with supporting students during clinical placements.

\section{THE INTERVENTION}

The process and content of the intervention, referred to as the REMARK programme (nuRse fEedback iMplementAtion framewoRK), is informed by the feedback principles and attributes critiqued in the literature, specifically, feedback principles to foster dialogic interactions. ${ }^{28}$ The intervention builds on prior work focussed on strategies to engage nurses in facilitating student learning and that encourage students to take responsibility for their learning. ${ }^{29} 30$

The intervention is shaped around the four constructs of NPT-coherence, cognitive participation, collective action and reflexive monitoring. These constructs are germane to how the researcher will approach, design and operationalise the practice-based intervention, as NPT recognises social factors are integral to the expression of agency. The agency of the student, buddy nurse and CF is fundamental to the adoption of the feedback and ultimately to the impact of the initiative.

\section{Coherence}

Coherence refers to meaning and sense-making. ${ }^{15}$ Creating a shared and meaningful understanding of feedback and its value is important to engage prospective participants. Preparation for the intervention is essential to optimise opportunities for the REMARK programme lead researcher to visit the clinical sites to inform and motivate students, buddy nurses and CFs to better engage with each other to share their understanding about feedback. It is important therefore to reach out to the leadership team at the intervention site where the workbased intervention will occur and discuss the best way to approach their teams. This includes conversations with the hospital management and the NUMs, considered 'gate-keepers' to unit areas, about demystifying feedback and the intention of the work-based intervention. Multiple visits will be important to liaise with these leadership groups about the best ways to gain access, 
for example, appropriate venues and times for sessions to optimise activities where the participants can openly and freely discuss what they understand to be feedback. Access to the study participants and the use of established communication channels-such as email notification, visual displays of key concepts (eg, posters, notes in staff allocation book), other printed resources and opportunities for conversations-facilitate coherence through collective discussions about the value, purpose and benefits of effective feedback.

\section{Cognitive participation}

Cognitive participation which refers to commitment and engagement ${ }^{15}$ is sought through dissemination of information and visibility of the programme modified in accordance with organisation structures and processes. Initially, interest in the provision of feedback processes for students will be explored with the buddy nurses during preparatory sessions. These sessions will raise awareness, understanding and expected obligations under the nurses' code of conduct about feedback. ${ }^{31}$ Preparatory sessions will be conducted 4 weeks prior to clinical placement blocks commencing at the intervention site-ideally four group sessions, one session per week, 30 min duration, session times/locations based on collaboration with the NUMs. Session topics will include: what is feedback, feedback attributes, challenges of feedback in the workplace/ clinical setting, the 3 'Cs' of feedback, the 2 min feedback conversation, formative workplace assessment and what is the impact of poor feedback. Commitment and engagement will be advanced through exploring participating nurses' interest and understanding of the topics and modifying the content and delivery accordingly.

\section{Collective action}

Collective action, which refers to effort and enactment ${ }^{15}$ and therefore relates to the range of accepted good practices known to engage buddy nurses and CFs in feedback with their student learners, will be established in delivery of the REMARK programme. These practices will include (but not limited to): formal inservice sessions, ad hoc small group and individual coaching, establishing needs of the study participants and what is practical in the healthcare setting. Participant engagement with any one of these methods will be referred to as a REMARK programme encounter. Although the primary focus will be introducing the buddy nurses and CFs to the REMARK programme, where possible, students will be invited to attend inservice sessions to provide an opportunity to encourage their open dialogue around feedback with the buddy nurses and CFs that support student learning in the clinical environment. Coherence and cognitive participation will continue throughout the programme facilitated through everyday communication channels and sessions by consistently highlighting simple techniques that participants can use to foster dialogue associated with effective feedback, such as increasing use of relevant open-ended questions.

Further to this, the norms of clinical practice-time pressured environments and the unpredictable nature of clinical demands-will shape the delivery of sessions. For example, sessions will be kept to 30 min duration, use 'quarantined' time allocated on existing scheduled unit inservice times (therefore not taking staff away from clinical care) and conducted on a weekly basis. However, when required, timing of sessions will be flexible. That is, where traditional unit inservice times may not be able to be accessed, alternative times/days will be negotiated with the NUMs or CFs.

Continuing reinforcement commensurate with collective action will be promoted through unit notices, meetings and emails advising of the presence of a 'feedback' coach (ie, the REMARK programme lead researcher). A number of small group or one-on-one structured, sequential activities, with the buddy nurses (refer table 2), CFs (refer table 3) and students (refer table 4) will be conducted by this feedback coach.

\begin{tabular}{|c|c|c|c|}
\hline Session & Intervention session topics & Activity & Duration \\
\hline 1 & $\begin{array}{l}\text { Purpose, benefit and impact of feedback } \\
\text { and attributes of effective feedback }\end{array}$ & $\begin{array}{l}\text { Participants to identify which attributes they use most/least } \\
\text { Discuss with group practical strategies to integrate less used } \\
\text { attributes in daily practice }\end{array}$ & $30 \mathrm{~min}$ \\
\hline 2 & $\begin{array}{l}\text { Feedback strategies - } 3 \text { Cs of feedback } \\
\text { (check-in; check-up; check-out) }\end{array}$ & $\begin{array}{l}\text { Group discussion to share what strategies participants currently } \\
\text { used to give students feedback } \\
\text { Individual activity to identify which attributes are used in each stage } \\
\text { of the } 3 \text { Cs strategy; share and discuss with group }\end{array}$ & $30 \mathrm{~min}$ \\
\hline 3 & Structuring feedback conversations & $\begin{array}{l}\text { Using the provided scenarios, write a feedback conversation } \\
\text { applying the discussed feedback technique; share and discuss with } \\
\text { group }\end{array}$ & $30 \mathrm{~min}$ \\
\hline 4 & Putting it all together & $\begin{array}{l}\text { In pairs use the feedback technique to give the student feedback } \\
\text { (and/or the clinicalfacilitator feedback) on their performance-try } \\
\text { to frame the feedback of student performance using professional } \\
\text { standards for practice; share and discuss with group }\end{array}$ & $30 \mathrm{~min}$ \\
\hline
\end{tabular}

REMARK, nuRse fEedback iMplementAtion framewoRK. 


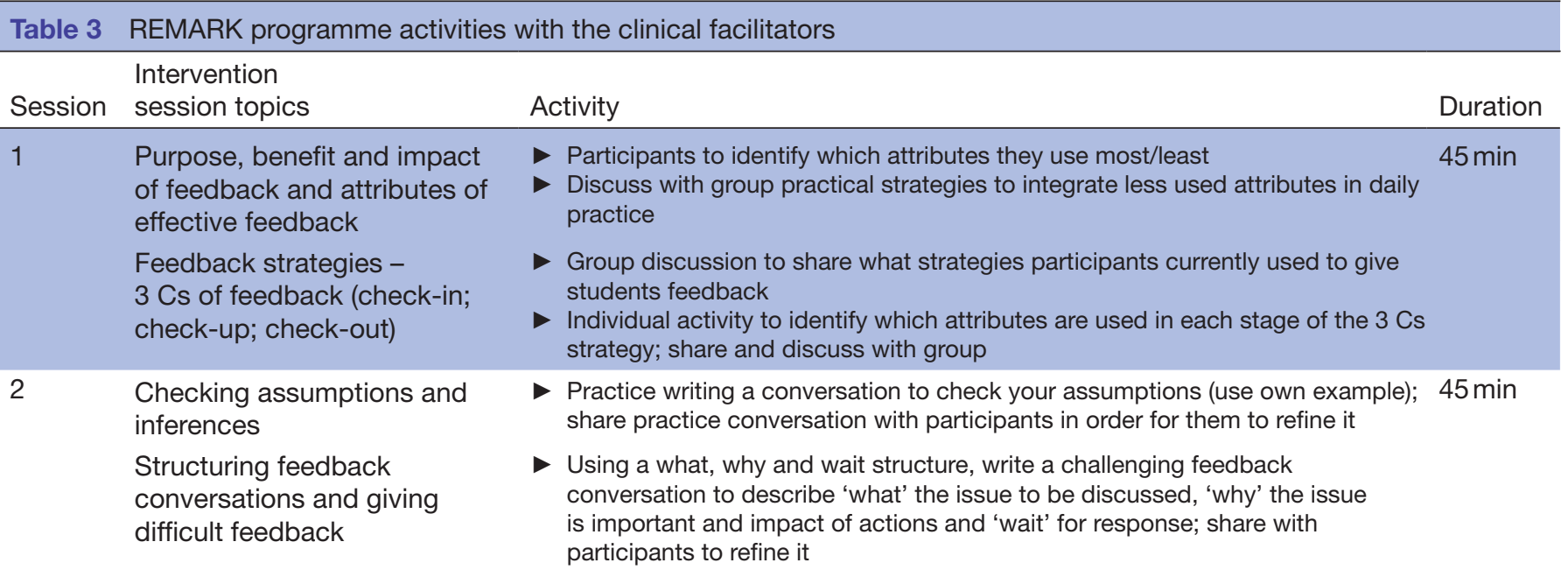

REMARK, nuRse fEedback iMplementAtion framewoRK.

\section{Reflexive monitoring}

The process of how participants appraise and comprehend feedback is referred to as reflexive monitoring. ${ }^{15}$ Encouraging and facilitating good feedback principles is warranted to assist the embedding of desired practices. Embedding feedback into work is shaped by factors that can inhibit or enable. Subsequent coaching with all study participants will be on an 'ad hoc' basis achieved by 'roving' through the clinical site. During periods of 'roving' the REMARK programme lead researcher will assist participants in self-reflection on the value of feedback and why or why not this occurred. These participants will be coached on how to draw on the enabling factors. Formal appraisal of implementation processes from the perspectives of the participants will be conducted at the end of the clinical placement period.

\section{Protocol adherence}

A component of robust intervention studies is consideration of the method and strategies that contribute to a greater certainty of the quality of a study's results. ${ }^{32} 33$ Protocol adherence focusses attention on the key elements within the study, such as the study design, intervention development and delivery and measuring the consistency in which the intervention is delivered as planned and accepted by the study participants. ${ }^{33-35}$ Strategies to enhance protocol adherence-and therefore reliability and validity of the study-include:

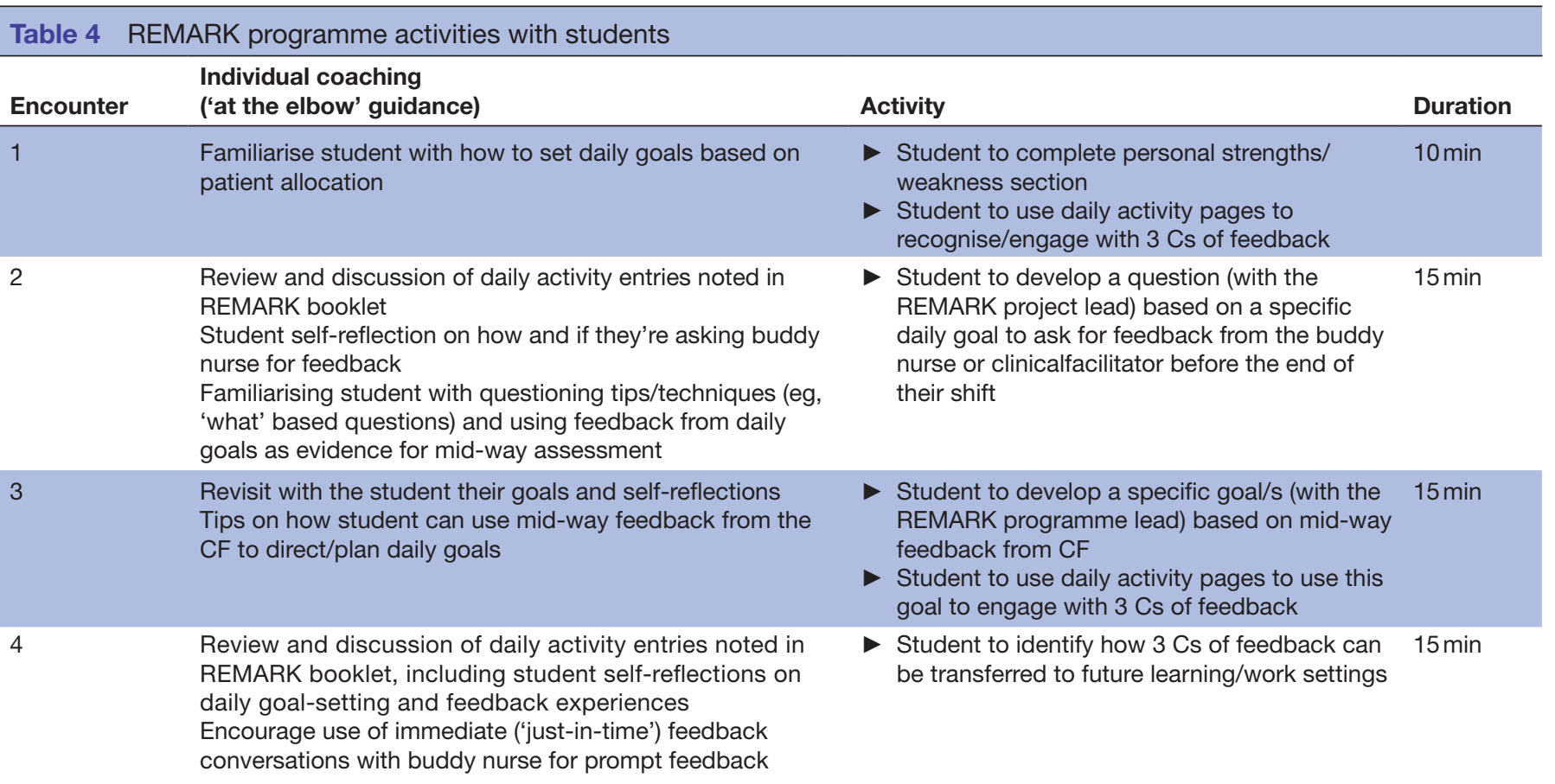

REMARK, nuRse fEedback iMplementAtion framewoRK. 
- Work-based intervention congruent with underpinning theory and current evidence;

- Use of validated measurement tools to evaluate study outcomes;

- REMARK programme lead researcher's experience with intervention studies and individual/group facilitation and coaching;

- Pre-engagement with key stakeholders and gatekeepers;

- Delivery of sessions by the REMARK programme lead researcher;

- Visibility of REMARK programme lead researcher in intervention units throughout study; and

- Comparable content and session length for all units at the intervention site.

\section{Intervention duration}

An intentional 8-week pre-engagement period at the intervention site will be undertaken by the REMARK programme lead researcher prior to the commencement of clinical placements. This period will facilitate an understanding of the context of the study environment and refine the intervention content to meet contextual and participant needs. A series of four, consecutive 4-week clinical placement blocks are provided by the participating university during the academic year. The REMARK programme will be run during each clinical placement block. While different students will be placed during the study period, it is possible that buddy nurses and clinical facilitators engaging in the REMARK programme will be consistent, thereby potentially increasing engagement and investment at the intervention site.

\section{DATA COLLECTION AND ANALYSIS}

\section{Outcomes and measurements}

Primary outcome

The primary outcome measure for the study will be the difference in undergraduate nursing student clinical performance assessment scores between control and intervention groups as measured by the ANSAT. What is of interest is whether student performance is optimised when the nursing team and students are continually facilitated and prompted to engage with good feedback practices. The ANSAT contains 23 items with each item scored on a scale from 1 to 5 , where a higher number indicates a higher standard of performance. ${ }^{36}$ A total score can be generated; the minimum score possible is 23 and the maximum score is 115 .

The ANSAT will be used to assess student performance on clinical placement and has been previously validated in a similar cohort. ${ }^{36}$ The ANSAT is also the authorised assessment tool within the undergraduate Bachelor of Nursing programme at the participating university. The tool will be completed by CFs as part of the university's standard assessment process and will be measured at three time points. ANSAT scores from the student's previous clinical placement is the baseline measure (pre-test). Mid-placement ANSAT scores contributes to the second data collection point (post-test 1), with the end-placement ANSAT scores contributing to the final data collection point (post-test 2). No additional time or training will be required to complete the ANSAT as this is a pre-established component of their role.

\section{Secondary outcomes (process data)}

To provide additional understanding of how all study participants experience feedback encounters during clinical placements, student, buddy nurse and CF perceptions will be evaluated at both control and intervention sites. Perceptions of feedback will be evaluated using the QFI specifically developed for this study. The practitioner QFI (for nurses and CFs) contains 46 items and the student QFI contains 50 items (online supplementary file 2). The items in both surveys are scored on a 5-point Likert scale ( $1=$ never to $5=$ always). These surveys will be administered to all participant groups at the end of each clinical placement block at both the control and the intervention sites. The inventories are based on key attributes of effective feedback derived from the literature. ${ }^{28}$ Information from the piloting of this inventory will be used to inform further psychometric testing.

Evaluation of participants' level of adoption of the REMARK programme at the intervention site will be measured by the NoMAD: It contains 20 items about implementation of the intervention (online supplementary file 3). The NoMAD asks participants' understanding, participation and action following implementation of the REMARK programme in the clinical unit. It is a validated, generic instrument developed to better understand the application and integration of complex interventions in healthcare from the view of those involved. ${ }^{35}$ The items have been adapted in accordance with guidelines ${ }^{35}$ to the intervention delivered in this study. The NoMAD will be administered to all participants at the end of each clinical placement block at the intervention site only.

Data collected from primary and secondary outcome measures will be non-identifiable and no personal identifying information will be collected. Figure 1 outlines the schema for data collection at each site. As data is nonidentifiable, the researchers will not be able to remove any outcome data collected from participants who withdraw from the study. All data will be presented as aggregate results. The REMARK programme lead researcher will be situated in the units for extended periods of time to be available to as many participants as possible. High visibility of REMARK programme lead researcher at the comparison site will facilitate immediacy of response to participants questions/inquiries.

\section{Sample size calculation}

The sample size required for the study has been calculated in relation to the primary outcome. According to formal calculations, a sample size of 139 participants is required at the control site and 70 participants at the intervention site to demonstrate a difference in students' 


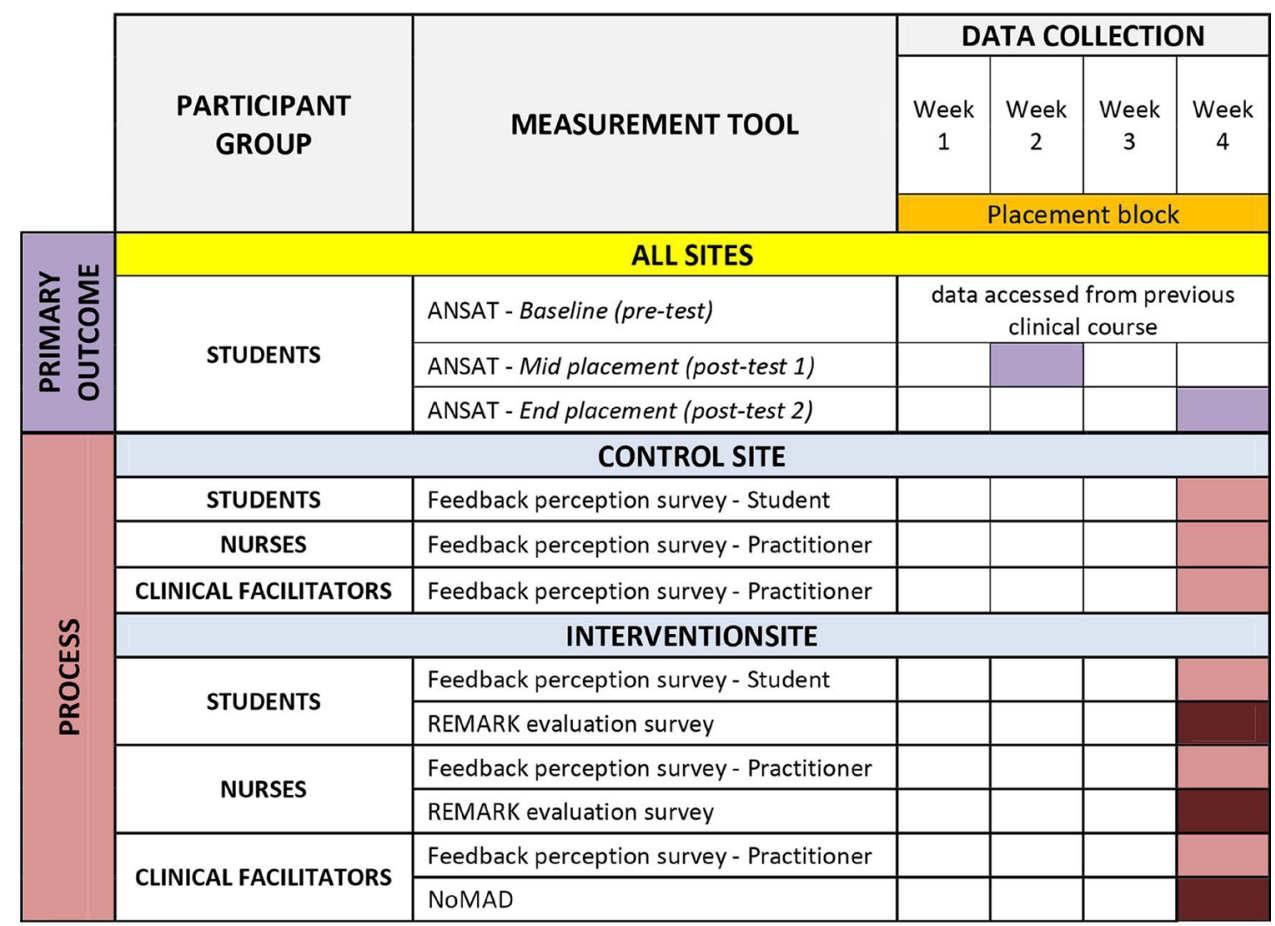

Figure 1 Data collection schema for participant groups at each site. ANSAT, Australian Nursing Standards Assessment Tool; REMARK,nuRse fEedback iMplementAtion framewoRK.

ANSAT scores at a confidence level of $95 \% .^{37}$ The sample size is adjusted to reflect an historic attrition rate of $5 \%$ from clinical placement. Historically the university places approximately 200 third-year students at the control site and approximately 80 third-year students at the intervention site. Therefore, the sample size calculated is a realistic target.

\section{Statistical analysis}

Data will be cleaned and examined for distribution and dispersion to assess distributional assumptions and relationships. Descriptive statistics including frequencies, percentages for categorical data and means, SD or medians and ranges for continuous data will be reported.

Total and mean ANSAT scores will be calculated per student at baseline and at their two assessment times. Assumptions regarding the relationships between variables will be scrutinised using a directed acyclic graph (DAG) ${ }^{38}$ Information from the DAG will inform direct and total effects requiring adjustment.

The effect of the intervention versus the control group on ANSAT scores will be assessed using a linear mixedeffects model (LMM) with random effects (of individual students) to compare and determine the effect of the intervention on student ANSAT scores across time, accounting for baseline ANSAT scores, student placement and change in time. Confounders, variables modifiers and biasses will be identified, eliminated where possible or appropriately dealt with thorough data transformation and the inclusion of interaction terms in the models.

Comparison of group means of the QFI will be performed by an independent t-test (or non-parametric equivalent Mann-Whitney U test). Analysis of variance (ANOVA) will be employed to test the differences in mean scores of the REMARK study for student, buddy nurse and clinical facilitator groups.

LMM and ANOVA are both robust tests where data are non-normal. ${ }^{39} 40$ The t-test is also fairly robust if data are non-normal if group size is greater than 40 and group size is roughly comparable. ${ }^{39}$

Missing data

Patterns of missing data will be explored. Sensitivity analyses will be conducted to determine the effects of missing data. If the effects of missingness is observed, appropriate imputation methods will be instituted. Linear mixedeffects models have the advantage of being flexible and accepting data in which some individuals do not have data at all time points and use all available data to provide estimates-not just complete cases. ${ }^{41}$

\section{DISCUSSION}

This protocol paper details the plans to improve the delivery of feedback between buddy nurses, CFs and third-year students when completing a 4-week clinical placement in a healthcare setting. The specific features of the intended REMARK programme have been developed to align with the NPT to promote the embedding of practices central to good feedback. Building the capacity of teams to improve feedback practices is important to enhance agility and responsiveness. While the value of feedback is routinely acknowledged there is limited evidence of how it can best be fostered and 
furthermore its contribution to enabling performance. Through the collection of data about the implementation process together with student workplace performance data at three time points, important insights on the use and impact of feedback in clinical settings can be gained. Any successes of the REMARK programme will be incorporated into future student and staff preparation for clinical placement ensuring equity for their ongoing development.

\section{Challenges}

The proposed activities commensurate with this implementation protocol face challenges common to other innovations in healthcare settings. Staff in these settings are routinely recognised as 'time-poor' and learning in the workplace is largely viewed as a secondary activity to the delivery of patient care. Additionally, the nature of shift work and the continual turnover of nurses (up to three times in the course of a day) provides a potential challenge to participation in the REMARK programme. The potential limited buy-in by the participants and/or attrition of the participants, is addressed through engagement with the local leaders, namely NUMs, who are very influential in shaping patterns of behaviour in the clinical units ${ }^{42}$ as well as the presence of the REMARK programme lead in the clinical units.

The results of this study will provide guidance not just in the value of good feedback processes but also the impact on student workplace performance. Evidence of the value of strategies to enhance behaviour and performance in clinical settings is limited. This proposed study has the potential to make a significant contribution to this literature.

\section{ETHICS, TIMELINES AND DISSEMINATION}

The study protocol (REMARK_Protocol_v1.1_20180314) was approved by Metro South Health Human Research Ethics Committee (HREC/18/QPAH/93) and Griffith University Human Research Ethics Committee (Reference number: 2018/341).

This study commenced on 23 July 2018. Data has been collected, with data entry and analysis yet to commence.

Dissemination of results will be local, national and international through forums, seminars, conferences and publications. Participants can contact the research team to discuss the findings of the study which will be in the form of aggregated results as data will be anonymous and non-identifiable to the researcher.

Correction notice This article has been corrected since it first published. The phrase 'REMARK evaluation survey' has been changed to "NoMAD" in section 'Secondary outcomes process data' and in figure 1.

Twitter Christine Ossenberg @COssenberg

Acknowledgements This research was undertaken as part of doctoral studies supported by Metro South Health Study, Education and Research Trust Account postgraduate scholarship and a Research Training Program Domestic Fee Offset scholarship provided by the Australian Government Department of Education and
Training. We gratefully acknowledge the detailed and invaluable statistical guidance provided by Dr Elizabeth Burmeister.

Contributors All authors were involved in developing the study design. $\mathrm{CO}$ is responsible for the intervention content and structure and will deliver all facets of the intervention. $\mathrm{CO}$ will be responsible for managing the data collection and analysis. $\mathrm{CO}$ prepared the initial manuscript with additional input by $\mathrm{AH}$. $\mathrm{AH}$ and MM critically reviewed the manuscript and suggested revisions. All authors read and approved the final manuscript.

Funding This study received funding from Metro South Hospital and Health Service through a competitive process. This source has no role in the design of the study or any role in the administration, analyses, interpretation of the data or decision to submit the manuscript for publication.

Competing interests None declared.

Patient consent for publication Not required.

Provenance and peer review Not commissioned; externally peer reviewed.

Open access This is an open access article distributed in accordance with the Creative Commons Attribution Non Commercial (CC BY-NC 4.0) license, which permits others to distribute, remix, adapt, build upon this work non-commercially, and license their derivative works on different terms, provided the original work is properly cited, appropriate credit is given, any changes made indicated, and the use is non-commercial. See: http://creativecommons.org/licenses/by-nc/4.0/.

ORCID iD

Christine Ossenberg http://orcid.org/0000-0001-8383-5043

\section{REFERENCES}

1 Yang $M$, Carless D. The feedback triangle and the enhancement of dialogic feedback processes. Teaching in Higher Education 2013;18:285-97.

2 Ramani S, Könings KD, Ginsburg S, et al. Feedback redefined: principles and practice. J Gen Intern Med 2019;34:744-9.

3 van der Leeuw RM, Teunissen PW, van der Vleuten CPM. Broadening the scope of feedback to promote its relevance to workplace learning. Acad Med 2018;93:556-9.

4 Carless D. Feedback loops and the longer-term: towards feedback spirals. Assess Eval High Educ 2019;44:705-14.

5 Killion J. The feedback process: Transforming feedback for professional learning. Oxford, Ohio: Learning Forward, 2015.

6 Boud D, Molloy E. Rethinking models of feedback for learning: the challenge of design. Assess Eval High Educ 2013;38:698-712.

7 Henderson A, Cooke M, Creedy DK, et al. Nursing students' perceptions of learning in practice environments: a review. Nurse Educ Today 2012;32:299-302.

8 Birken SA, Powell BJ, Shea CM, et al. Criteria for selecting implementation science theories and frameworks: results from an international survey. Implement Sci 2017;12:124.

9 Rabin BA, Brownson RC, Haire-Joshu D, et al. A glossary for dissemination and implementation research in health. $J$ Public Health Manag Pract 2008;14:117-23.

10 Nilsen P. Making sense of implementation theories, models and frameworks. Implement Sci 2015;10:53.

11 Foy R, Ovretveit J, Shekelle PG, et al. The role of theory in research to develop and evaluate the implementation of patient safety practices. BMJ Qual Saf 2011;20:453-9.

12 Eccles MP, Armstrong D, Baker R, et al. An implementation research agenda. Implementation Sci 2009;4.

13 Tabak RG, Khoong EC, Chambers DA, et al. Bridging research and practice: models for dissemination and implementation research. Am $J$ Prev Med 2012;43:337-50.

14 May CR, Mair F, Finch T, et al. Development of a theory of implementation and integration: normalization process theory. Implement Sci 2009;4:29.

15 May C, Finch T. Implementing, embedding, and integrating practices: an outline of normalization process theory. Sociology 2009;43:535-54.

16 May CR, Cummings A, Girling M, et al. Using normalization process theory in feasibility studies and process evaluations of complex healthcare interventions: a systematic review. Implement Sci 2018;13:80.

17 Finch TL, Rapley T, Girling M, et al. Improving the normalization of complex interventions: measure development based on normalization process theory (NoMAD): study protocol. Implement Sci 2013;8:43. 
18 Murray E, Treweek S, Pope C, et al. Normalisation process theory: a framework for developing, evaluating and implementing complex interventions. BMC Med 2010;8:63.

19 Glynn LG, Glynn F, Casey M, et al. Implementation of the smart move intervention in primary care: a qualitative study using normalisation process theory. BMC Fam Pract 2018;19:48. 1.

20 O'Donnell CA, Mair FS, Dowrick C, et al. Supporting the use of theory in cross-country health services research: a participatory qualitative approach using normalisation process theory as an example. BMJ Open 2017;7:e014289.

21 de Brún T, O'Reilly-de Brún M, O'Donnell CA, et al. Learning from doing: the case for combining normalisation process theory and participatory learning and action research methodology for primary healthcare implementation research. BMC Health Serv Res 2016;16:346.

22 Merton RK. Social theory and social structure. New York: The Free Press, 1968.

23 Walker R, Cooke M, McAllister M. The meaningful experiences of being a registered nurse (RN) Buddy. Nurse Educ Today 2008;28:760-7.

24 Curtis E, Drennan J. Quantitative health research: issues and methods. McGraw-Hill Education 2013.

25 Babbie ER. The practice of social research. 14. Boston, MA Cengage Learning, 2016.

26 Burns RB. Introduction to research methods. 4. London: SAGE publications, 2000

27 Polit DF, Beck CT. Nursing research: Principles and methods. 7. Philadelphia: Lippincott Williams \& Wilkins, 2004.

28 Ossenberg C, Henderson A, Mitchell M. What attributes guide best practice for effective feedback? A scoping review. Adv Health Sci Educ Theory Pract 2019;24:383-401.

29 Cooke M, Walker R, Creedy D, et al. Clinical progression Portfolio: a resource for enhancing learning partnerships. Nurse Educ Pract 2009;9:398-402.

30 Henderson A, Harrison P, Rowe J, et al. Students take the lead for learning in practice: a process for building self-efficacy into undergraduate nursing education. Nurse Educ Pract 2018;31:14-19.
31 Nursing and Midwifery Board of Australia. Code of conduct for nurses, 2018. Available: https://www.nursingmidwiferyboard.gov. au/Codes-Guidelines-Statements/Professional-standards.aspx [Accessed Mar 2019].

32 Bellg AJ, Borrelli B, Resnick B, et al. Enhancing treatment fidelity in health behavior change studies: best practices and recommendations from the NIH behavior change Consortium. Health Psychol 2004;23:443-51.

33 Bova C, Jaffarian C, Crawford S, et al. Intervention fidelity: monitoring drift, providing feedback, and assessing the control condition. Nurs Res 2017;66:54-9.

34 Resnick B, Inguito P, Orwig D, et al. Treatment fidelity in behavior change research: a case example. Nurs Res 2005;54:139-43.

35 Finch TL, Girling M, May CR, et al. Improving the normalization of complex interventions: part 2 - validation of the NoMAD instrument for assessing implementation work based on normalization process theory (NPT). BMC Med Res Methodol 2018;18:135.

36 Ossenberg C, Mitchell M, Henderson A. Adoption of new practice standards in nursing: revalidation of a tool to measure performance using the Australian registered nurse standards for practice. Collegian 2019

37 Raosoft Inc. Sample size calculator, 2004. Available: http://www. raosoft.com/samplesize.html [Accessed Mar 2019].

38 Textor J. DAGitty - draw and analyze causal diagrams, 2020. Available: http://www.dagitty.net/ [Accessed Feb 2020]

39 Polit DF. Statistics and data analysis for nursing research. 2nd. Upper Saddle River; New Jersey: Pearson, 2010.

40 Saefken B, Kneib T, van Waveren C-S, et al. A unifying approach to the estimation of the conditional Akaike information in generalized linear mixed models. Electron J Stat 2014;8:201-25.

$41 \mathrm{Xu} \mathrm{S}$, Blozis SA. Sensitivity analysis of mixed models for incomplete longitudinal data. J Educ Behav Stat 2011;36:237-56.

42 Henderson A, Burmeister L, Schoonbeek S, et al. Impact of engaging middle management in practice interventions on staff support and learning culture: a quasi-experimental design. J Nurs Manag 2014;22:995-1004 